\title{
Cuidado Ambiental em Tempos de Sustentabilidade: Relação Entre Compromisso Pró-Ecológico e Orientação de Futuro
}

\author{
Raquel F. Diniz \\ José Q. Pinheiro \\ Universidade Federal do Rio Grande do Norte \\ Natal, RN, Brasil
}

\begin{abstract}
RESUMO
A fim de explorar algumas das dimensões psicológicas que fazem parte de estilos de vida sustentáveis, no presente estudo foram investigadas relações entre indicadores de compromisso pró-ecológico (escala de Ambientalismos Ecocêntrico e Antropocêntrico) e de orientação temporal (escala de Consideração de Consequências Futuras) e suas associações com o relato espontâneo de práticas de cuidado ambiental. Participaram 380 estudantes universitários, a maioria do sexo feminino $(77 \%)$, e com média de idade de 22 anos $(D P=3,66)$. Os resultados foram coerentes com estudos anteriores, ainda que com índices estatísticos modestos. Foi corroborada a associação entre compromisso pró-ecológico, mais fortemente o ecocentrismo, e a orientação temporal de futuro. Houve associações evidentes entre o antropocentrismo, a apatia ambiental e o imediatismo, e a ausência de relatos de práticas de cuidado ambiental. São discutidas as implicações e limitações do estudo, assim como são feitos apontamentos para estudos futuros.
\end{abstract}

Palavras-chave: Psicologia ambiental; atitudes; desenvolvimento sustentável; perspectiva de tempo.

\section{ABSTRACT}

Environmental Care in Times of Sustainability: The Relationship Between Pro-Environmental Commitment and Future Orientation

In order to explore some of the psychological dimensions that are part of a sustainable lifestyle, in the present research were investigated relationships between indicators of pro-ecological commitment (Ecocentric and Anthropocentric Environmentalism scales) and temporal orientation (Consideration of Future Consequences scale), and their associations with self-reported practices of environmental care. 380 college students participated, most were female $(77 \%)$, with a mean age of 22 years old $(\mathrm{SD}=3.66)$. The results were consistent with previous studies, albeit with modest statistical indices. The association between pro-ecological commitment, stronger in case of ecocentrism, and future time orientation was corroborated. There were clear associations between anthropocentrism, environmental apathy and immediacy, and the absence of reports of environmental care practices. The implications and limitations of the study are discussed, as well are made appointments for future researches.

Keywords: Environmental psychology; attitudes; sustainable development; time perspective.

\section{RESUMEN}

Cuidado Ambiental en Tiempos de Sostenibilidad: Relación Entre Compromiso Proecológico y Orientación de Futuro Con el fin de explorar algunas de las dimensiones psicológicas que forman parte de los estilos de vida sostenibles, en ese trabajo fueron investigadas las relaciones entre indicadores del compromiso pro-ecológico (escala de Ambientalismos Ecocéntrico y Antropocéntrico) y de la orientación temporal (escala de Consideración de Consecuencias Futuras) y sus asociaciones con el relato espontáneo de prácticas de cuidado ambiental. Participaron 380 estudiantes de grado, la mayoría del sexo femenino (77\%), y con media de edad de 22 años $(\mathrm{DP}=3,66)$. Los resultados fueron coherentes con los de trabajos anteriores, aunque con índices estadísticos modestos. Fue corroborada la asociación entre el compromiso pro-ecológico, más fuerte con el ecocentrismo, y la orientación temporal de futuro. Hubo asociaciones evidentes entre el antropocentrismo, la apatía ambiental y el inmediatismo, y la ausencia de relatos de prácticas de cuidado con el medio ambiente. Son discutidas las implicaciones y limitaciones de la investigación, así como fueron hechos apuntes para futuras investigaciones.

Palabras clave: Psicología ambiental; actitudes; desarrollo sostenible; perspectiva de tiempo. 


\section{INTRODUÇÃO}

O interesse pela questão ambiental vem se consolidando ao longo das últimas quatro décadas em diversos âmbitos, tendo adquirido novos contornos a partir da emergência do conceito de sustentabilidade. Definida como o modelo de desenvolvimento que "atende às necessidades do presente sem comprometer a possibilidade das gerações futuras atenderem as suas próprias necessidades" (Comissão Mundial sobre Meio Ambiente e Desenvolvimento, 1991, p. 46), a partir dela se depreende o desafio para as gerações atuais em adotar estilos de vida que prezem pela manutenção dos recursos em função, também, do bem-estar das próximas gerações. Assim, a sustentabilidade demanda a ampliação do foco dado à compreensão das interações humano-ambientais, visto que contempla uma dimensão temporal expressa no princípio da solidariedade intergeracional, que enfatiza a importância da adoção de um estilo de vida austero e assegura a igualdade entre as gerações em sua relação com o sistema natural (Pol, 2002).

Dentre as diversas disciplinas que se dedicam ao estudo de tais interações está a Psicologia Ambiental. Sua interface com a sustentabilidade tem sido discutida por diversos autores que afirmam a importância de sua contribuição para a construção do conhecimento sobre as relações pessoa-ambiente. Tal conhecimento pode fornecer bases para intervenções e mudanças comportamentais mais consistentes e duradouras, promovendo estilos de vida orientados por princípios alternativos ao modelo de desenvolvimento vigente nas sociedades ocidentais e de economia capitalista (Corral-Verdugo, 2010; García-Mira \& Marcote, 2009; Kazdin, 2009).

No âmbito da pesquisa psicológica, os estilos de vida sustentáveis foram definidos por Corral-Verdugo e Pinheiro (2004) como "conjunto de ações efetivas, deliberadas e antecipadas que resultam na preservação dos recursos naturais, incluindo a integridade das espécies animais e vegetais, assim como o bem estar individual e social das gerações atuais e futuras" (p. 10). Trata-se de um conceito multidimensional, que comporta diversas dimensões psicológicas que podem estar associadas entre si.

A fim de mais bem compreender e delimitar os avanços das investigações sobre tais dimensões no âmbito da psicologia, Corral-Verdugo (2010) as reuniu em três eixos, quais sejam: variáveis disposicionais (antecedentes), comportamento, e repercussões psicológicas (consequentes). No presente estudo são abordadas as relações entre duas variáveis disposicionais - o compromisso pró-ecológico e a orientação temporal de futuro - e a associação das mesmas com relatos de práticas de cuidado ambiental (dimensão comportamental).

\section{COMPROMISSO PRÓ-ECOLÓGICO}

As investigações sobre os determinantes dos comportamentos de cuidado ambiental, em geral, abordam associações e relações entre diversas variáveis disposicionais - como crenças, visões de mundo, normas, atitudes, valores - que embasam modelos explicativos. Tais investigações têm sinalizado sistematicamente o caráter preditivo ou mediador de tais variáveis para o comportamento (e.g., CorralVerdugo, 2010; Dietz, Fitzgerald, \& Shwom, 2005; Groot \& Steg, 2010; Pato \& Tamayo, 2006; Pinheiro \& Pinheiro, 2007).

A fim de sintetizar o entendimento sobre a postura favorável ao meio ambiente, Gurgel e Pinheiro (2011) nomearam compromisso pró-ecológico (CPE) “a relação cognitiva e/ou afetiva, de caráter positivo, que as pessoas estabelecem com o meio ambiente ou parte do mesmo, responsabilizando-se e interessando-se por ele" (p. 159). Tal definição resulta também do interesse dos autores em lançar mão de uma linguagem mais acessível às diferentes áreas afins, como a educação ambiental, visto tratar-se de uma nomenclatura mais clara e próxima à linguagem comum.

No âmbito do conhecimento sobre o compromisso pró-ecológico estão as noções de ecocentrismo e antropocentrismo (Thompson \& Barton, 1994); ambas compreendidas como posturas favoráveis ao meio ambiente, que contemplam o interesse pela questão ambiental e manutenção dos recursos naturais, mas diferem quanto às motivações e bases valorativas. Pessoas ecocêntricas reconhecem a importância da manutenção dos recursos naturais independentemente da situação econômica ou qualidade de vida humana e para elas a natureza tem uma dimensão espiritual e valor intrínseco que é refletida em afetos e experiências em ambientes naturais. Numa postura diversa, pessoas antropocêntricas são favoráveis à manutenção dos recursos naturais em função do conforto e qualidade de vida humana, reconhecendo que a saúde depende da preservação dos recursos e de um ecossistema equilibrado.

A fim de explorar as diferenças entre tais posturas, Thompson e Barton (1994) desenvolveram um instrumento com escalas de mensuração do antropocentrismo e do ecocentrismo (12 itens cada). Foi acrescida uma escala de apatia ambiental ( 9 itens) como contraponto às escalas anteriores. As autoras observaram que ecocêntricos expressaram menor apatia ambiental 
e apresentaram maior tendência a conservar os recursos naturais e participar de organizações ambientais. Tal postura teria então como base valores biosféricos. Por outro lado, antropocêntricos apresentaram maior pontuação na apatia ambiental, menor intenção de atuar de forma pró-ecológica e menor interesse em participar de organizações ambientais. Nesse caso, teriam como base valores egoístas e de altruísmo social.

Posteriormente, num estudo multinacional, Schultz e Zelezny (1999) utilizaram uma versão reduzida do instrumento, composta apenas por itens das escalas de antropocentrismo e ecocentrismo, descartando a escala de apatia ambiental. Os autores reportaram resultados semelhantes aos do estudo original, porém ressaltaram as limitações encontradas nas associações entre as escalas de ecocentrismo e antropocentrismo e o relato de ações pró-ecológicas. Em estudo com estudantes brasileiros, Coelho, Gouveia e Milfont (2006) utilizaram a versão reduzida das escalas e corroboraram os resultados dos estudos anteriores, inclusive as limitações das associações com os relatos de práticas. No desenvolvimento de um inventário de atitudes ambientais, Milfont e Duckitt (2010) reuniram diferentes escalas propostas pela literatura e, como nos estudos anteriores, incluíram apenas itens das escalas de ecocentrismo e antropocentrismo, desconsiderando a escala da apatia ambiental.

Em seu estudo com estudantes universitários brasileiros, Pinheiro e Pinheiro (2007) utilizaram a versão completa da escala e identificaram associações significativas entre a ausência de relato de comportamento pró-ecológico e a apatia ambiental, corroborando os achados do estudo original das autoras da escala. Com base nesse e em outros estudos posteriores desenvolvidos em nosso grupo de pesquisa (Azevedo, 2008, Souza, 2009), na presente investigação foi utilizada uma adaptação da versão completa do instrumento de Thompson e Barton (1994), assim como o recurso metodológico ao termo cuidado ambiental, para tratar do relato espontâneo de práticas avaliadas como ambientalmente significativas, como será exposto a seguir.

\section{ORIENTAÇÃO TEMPORAL}

Com o reconhecimento da dimensão temporal explícita no conceito de sustentabilidade, em momento mais recente, um número crescente de estudos tem se debruçado sobre suas associações com o compromisso pró-ecológico, apresentando evidências empíricas que justificam seu enquadramento no escopo de determinantes dos estilos de vida sustentável (e.g., Arnocky, Milfont, \& Nicol, 2013; Pinheiro \& Corral-
Verdugo, 2010; Gifford et al., 2009; Joireman, 2005; McElwee \& Brittain, 2009; Milfont \& Gouveia, 2006).

De acordo com Pinheiro (2006), há pelo menos duas teorizações sobre temporalidade de interesse direto para o entendimento dos estilos de vida sustentáveis. De acordo com Zimbardo e Boyd (1999), a perspectiva temporal constitui um processo fundamental para o funcionamento tanto individual como social. Trata-se da organização, em termos de categorias temporais (passado, presente e futuro), que ajuda a conferir sentido e ordem às vivências pessoais. Segundo os autores, as perspectivas temporais aprendidas exercem uma influência dinâmica sobre muitos julgamentos, decisões importantes e ações.

A segunda teorização, a noção de consideração de consequências futuras (CCF - Strathman, Gleicher, Boninger, \& Edwards, 1994), expressa o quanto a pessoa considera as consequências futuras das ações praticadas no presente e a influência sobre as escolhas comportamentais. Envolve o conflito intrapessoal entre o comportamento presente com o conjunto de resultados imediatos e o conjunto de resultados futuros. De acordo com Joireman (2005), as diferenças individuais podem influenciar a percepção das consequências de uma ação e desta forma direcionam e/ou influenciam a sensibilidade em relação ao quão imediata ou distante serão percebidas estas consequências.

O instrumento desenvolvido por Strathman et al. (1994) como indicador de CCF foi composto originalmente por cinco itens com redação positiva (e.g., "Eu penso sobre como as coisas podem vir a ser no futuro, e tento influenciá-las com minhas ações do dia-a-dia") e outros sete com redação negativa, que deveriam ter seus escores revertidos no processo de análise (e.g., "Eu só faço coisas para atender meus interesses imediatos, pois o futuro será o que tiver de ser"). Com base nessa estrutura unidimensional, a baixa pontuação na escala estaria associada à priorização das necessidades imediatas, e a alta pontuação expressaria preocupação pelo futuro, e as consequências das ações presentes seriam utilizadas pelo respondente como guias para escolha comportamental (Strathman et al., 1994).

Estudos posteriores têm questionado a estrutura original desta escala, propondo uma redução do número de itens (Petrocelli, 2003), e apresentado evidências de uma estrutura bifatorial, mediante a conformação de dois fatores (ou construtos) subjacentes à escala de CCF. De acordo com Joireman, Balliet, Sprott, Spangenberg e Schultz (2008), o primeiro fator representa o interesse pelas consequências imediatas do comportamento atual e abrange os sete itens de redação negativa (imediatismo), já o segundo fator expressa o interesse pelas consequências distantes no futuro e abrange os 
itens positivos (futuro), resultados corroborados por estudos posteriores (Joireman, Shaffer, Balliet, \& Strathman, 2012; Rappange, Brouwer, \& Van Exel, 2009; Toepoel, 2010).

Investigações que abordaram as associações entre indicadores do compromisso ecológico e as escalas de imediatismo e futuro também apontaram a pertinência da estrutura bifatorial como via para aclarar as associações entre o CPE e a orientação de futuro (Arnocky, Milfont, \& Nicol, 2013; Barros, 2011; Enzler, 2013). Em uma meta-análise quantitativa dos estudos desenvolvidos nos últimos anos sobre as associações entre indicadores de compromisso próecológico e perspectiva temporal, Milfont, Wilson e Diniz (2012) demonstraram que a perspectiva temporal, como uma variável disposicional, exerce influência sobre as atitudes pró-ecológicas e sobre o comportamento pró-ecológico em geral. Os autores demonstraram ainda que a orientação temporal de futuro apresenta um pequeno efeito sobre as atitudes ambientais e médio efeito sobre o engajamento em ações ambientais (dimensão comportamental).

Com base no exposto, o presente estudo teve como objetivo investigar as associações entre duas variáveis disposicionais de estilos de vida sustentáveis, compromisso pró-ecológico e orientação temporal, assim como as relações entre seus indicadores e o relato de práticas de cuidado ambiental.

\section{MÉTODO}

\section{Participantes}

Participaram voluntariamente dessa pesquisa 380 estudantes de cursos universitários, de uma instituição pública de ensino superior da cidade do Natal (RN), selecionados segundo a técnica de amostragem por conveniência. A maioria era do sexo feminino (77\%), estudou em escolas particulares antes de ingressar na universidade $(68 \%)$ e a média de idade foi de 22 anos $(D P=3,66$; amplitude de 17 a 44).

\section{Instrumento}

O questionário desenvolvido para a pesquisa foi dividido em duas partes. A primeira contemplou dados sociodemográficos (sexo, idade, tipo de escola que estudou por mais tempo) e uma questão dicotômica (Sim/Não) relativa à prática pessoal de cuidado ambiental. Em caso de resposta afirmativa, o participante deveria relatar suas ações que avaliasse como sendo de cuidado ambiental, tendo sido considerados cuidadores os participantes que fizeram esse relato. Caso não fosse relatado algum tipo de ação, o participante seria considerado não-cuidador.
A segunda parte do questionário compreendeu a Escala de Ambientalismos Ecocêntrico e Antropocêntrico (AEA - Thompson \& Barton, 1994), como indicador do compromisso pró-ecológico. Tal como na versão original, os 33 itens da escala abordaram antropocentrismo (e.g., "A razão mais importante pela qual devemos conservar a natureza é a sobrevivência dos seres humanos"), ecocentrismo (e.g., "A natureza deve ser valorizada por si mesma") e apatia ambiental (e.g., "Ameaças ambientais tais como o desmatamento e a diminuição da camada de ozônio têm sido exageradamente divulgadas"). Os participantes indicaram seu grau de concordância ou discordância numa escala tipo Likert, variando de $1=$ Discordo muito a $7=$ Concordo muito.

O questionário compreendeu ainda, como indicador da orientação temporal, a Escala de Consideração de Consequências Futuras (CCF - Strathman et al., 1994), considerando sua estrutura bifatorial, com itens de orientação imediatista (e.g., "Eu só faço coisas para atender meus interesses imediatos, pois o futuro será o que tiver de ser") e de orientação de futuro (e.g., "Eu penso sobre como as coisas podem vir a ser no futuro, e tento influenciá-las com minhas ações do dia-a-dia"). Novamente, foi utilizada uma escala de resposta tipo Likert com sete pontos, de $1=$ Bastante inaplicável a 7=Bastante aplicável. Ambas as escalas utilizadas foram submetidas à validação semântica em estudos anteriores com populações de características semelhantes, os quais possibilitaram adaptações aos grupos investigados e orientaram a adoção de escalas Likert de sete pontos, a fim de favorecer maior variabilidade das respostas (Azevedo, 2008; Pinheiro \& Pinheiro, 2007; Sousa, 2009).

\section{Procedimentos}

A aplicação dos questionários foi feita em salas de aula, mediante autorização prévia da instituição e do professor responsável pela turma, supervisionada por um único e mesmo pesquisador, adotando um procedimento padrão, com duração máxima de 30 minutos. Ressalta-se que a pesquisa foi aprovada pelo Comitê de Ética em Pesquisa do Hospital Universitário Onofre Lopes (CEP-HUOL/UFRN). Nesse sentido, foram considerados aspectos éticos que incluíram informe livre e esclarecido aos participantes sobre os objetivos da pesquisa, possíveis riscos envolvidos e compromisso em manter total confidencialidade dos dados de identificação dos mesmos.

\section{Análise dos dados}

Ademais das análises descritivas, a fim de identificar as condições de distribuição das variáveis 
específicas para o grupo investigado, foi utilizada a análise fatorial exploratória. Em função da dimensão do conjunto de dados e com vistas a alcançar maior porcentagem de variância explicada, uma estrutura mais clara e com melhores índices estatísticos, foi utilizada uma extração de componentes principais (ACP). Na composição dos fatores, foram eliminados os itens com cargas fatoriais inferiores a 0,4 ou cargas satisfatórias em mais de um fator. Com as novas variáveis, criadas a partir da média ponderada para cada fator, foram feitas análises de regressão linear a fim de verificar o sentido e a magnitude das relações entre os construtos (Dancey \& Reidy, 2006).

Os relatos das ações de cuidado ambiental foram digitalizados e submetidos à análise de conteúdo (Sommer \& Sommer, 1997). Para tanto, foram estabelecidas macro categorias prévias, definidas com base em estudos anteriores, quais sejam: manejo de resíduos (e.g. separação ou reaproveitamento dos resíduos, coleta seletiva), conscientização (educação ambiental, participação em eventos e projetos), consumo (e.g. economia de recursos, produtos ecológicos), preservação (e.g.cultivo de plantas) e formação (pesquisa, curso) (Barros, 2011; Link, 2006; Pinheiro \& Pinheiro, 2007).

\section{RESULTADOS}

Inicialmente são apresentados os resultados das análises das escalas, suas correlações e modelos explicativos e, em seguida, as associações com o relato da prática de cuidado ambiental. A distribuição das variáveis da escala de Ambientalismos Ecocêntrico e Antropocêntrico (AEA) foi semelhante à encontrada por Thompson e Barton (1994), com a exclusão de doze itens e total de $39,98 \%$ da variância explicada $\left(\mathrm{KMO}=0,78 ; X^{2}=1657,266 ; d f=210 ; p<0,001\right)$.

O Fator "Ecocentrismo" contemplou o afeto positivo relacionado com o contato direto com a natureza (e.g., "Preciso passar algum tempo junto à natureza para ser feliz"; "Quando me sinto triste encontro conforto na natureza.”). Os itens excluídos abordavam posicionamentos favoráveis à proteção de áreas naturais, assim como afetos negativos relacionados com o desmatamento, o que aponta para uma reconfiguração da postura ecocêntrica no grupo investigado. Já o Fator "Antropocentrismo", contemplou uma postura em favor da manutenção dos recursos naturais para a manutenção da qualidade de vida e do bem-estar humano (e.g., "Precisamos preservar os recursos para manter uma alta qualidade de vida"; "A razão mais importante para a conservação ambiental é a sobrevivência humana"). Os dois itens excluídos contemplavam a diferenciação entre seres humanos e demais seres vivos. Por fim, o Fator "Apatia Ambiental" (e.g., "Eu acho que o problema do esgotamento dos recursos naturais não é tão ruim como se diz"; "A maioria dos problemas ambientais se resolverá por conta própria se lhes for dado tempo suficiente"), contemplou o não envolvimento com a questão ambiental, e teve três itens excluídos. Embora o último fator tenha apresentado índices estatísticos inferiores aos demais, apresentou correlações significativas nas análises bivariadas.

Quanto à escala de Consideração de Consequências Futuras (CCF), na conformação da estrutura bifatorial não houve exclusão de itens, tendo explicado 41,65\% da variância total $\left(\mathrm{KMO}=0,821 ; X^{2}=855,523\right.$; $d f=66 ; p<0,001)$. O Fator Imediatismo foi composto pelos itens que tratam da orientação temporal focada no momento presente (e.g., "Eu só faço coisas para atender meus interesses imediatos, pois o futuro será o que tiver de ser"; "Eu acho que se sacrificar agora é em geral desnecessário já que se pode lidar com acontecimentos futuros em um momento posterior."). Já o Fator Futuro agrupou os itens relativos à consideração de consequências futuras (e.g., a "Eu estou disposto a sacrificar minha felicidade ou bem-estar imediatos a fim de alcançar consequências futuras"; "É comum eu me envolver em alguma ação para conseguir resultados que podem demorar muitos anos a aparecer"). Os índices estatísticos das escalas são apresentados na Tabela 1.

TABELA 1

Número de itens, médias, desvios padrão e Alfas de Cronbach das escalas AEA e CCF

\begin{tabular}{|c|c|c|c|c|c|}
\hline Escala & $N^{o}$ de itens & $M$ & s.d. & $\begin{array}{c}\text { \% Variância } \\
\text { explicada }\end{array}$ & $\begin{array}{c}\text { Alfa de } \\
\text { Cronbach }\end{array}$ \\
\hline \multicolumn{6}{|c|}{ Ambientalismos Ecocêntrico e Antropocêntrico } \\
\hline Antropocentrismo & 10 & 3,76 & 0,95 & 15,59 & 0,75 \\
\hline Ecocentrismo & 5 & 5,05 & 1,25 & 13,89 & 0,78 \\
\hline Apatia ambiental & 6 & 2,49 & 0,94 & 10,49 & 0,59 \\
\hline \multicolumn{6}{|c|}{ Consideração de Consequências Futuras } \\
\hline Imediatismo & 7 & 1,44 & 0,60 & 25,12 & 0,75 \\
\hline Futuro & 5 & 2,94 & 0,56 & 16,03 & 0,58 \\
\hline
\end{tabular}


As correlações entre ecocentrismo, antropocentrismo e apatia ambiental e as orientações temporais, imediatismo e futuro, embora tendo alcançado índices de fracos a moderados, foram significativas e nas direções esperadas. De forma mais evidente e delimitando extremos, o Ecocentrismo apresentou correlação positiva com futuro e a apatia ambiental teve correlação positiva com o imediatismo. Já o antropocentrismo apresentou correlação positiva com ambas as orientações temporais (Tabela 2).

TABELA 2

Correlações entre antropocentrismo, ecocentrismo, apatia ambiental e orientação temporal

\begin{tabular}{lcccc}
\hline \multicolumn{1}{c}{ Fatores } & 1 & 2 & 3 & 4 \\
\hline 1. Antropocentrismo & - & & & \\
2. Ecocentrismo & 0,03 & - & & \\
3. Apatia Ambiental & $0,12^{*}$ & $-0,15^{*}$ & - & \\
4. Imediatismo & $0,22^{*}$ & $-0,22^{*}$ & $0,40^{*}$ & - \\
5. Futuro & $0,12^{*}$ & $0,33^{*}$ & $-0,18^{*}$ & $-0,30 *$ \\
\hline
\end{tabular}

$* p<0,05$ (bicaudal).

A partir das análises de regressão foi possível avançar no entendimento sobre a direção das relações entre os fatores das escalas. Na primeira análise, com os fatores da escala AEA colocados no primeiro passo, foram encontradas três equações significativas: ecocentrismo $[\mathrm{F}(1,367)=55,35 ; p<0,001 ; \mathrm{R}=0,36)]$, teve como preditor o futuro $(\beta=0,36 ; p<0,001)$; antropocentrismo $[\mathrm{F}(2,364)=21,58 ; p<0,001$; $\mathrm{R}=0,32)$ ], teve como preditores tanto o imediatismo $(\beta=0,31 ; p<0,001)$ quanto o futuro $(\beta=0,24 ; p<0,001)$; e a apatia ambiental $[\mathrm{F}(1,364)=50,92 ; p<0,001$; $\mathrm{R}=0,35)$ teve como preditor o imediatismo $(\beta=0,39$; $p<0,001)$. A porcentagem de variância explicada para os modelos $\left(\mathrm{R}^{2}\right)$ foi de $8 \%$ a $12 \%$.

$\mathrm{Na}$ segunda análise, com a ordem de entrada dos fatores invertida, o imediatismo $[\mathrm{F}(3,359)=33,67$; $p<0,001 ; \mathrm{R}=0,47)]$ teve como preditores a apatia ambiental $(\beta=0,34 ; p<0,001)$, o antropocentrismo ( $\beta=0,19 ; p<0,001)$, e negativamente o ecocentrismo $(\beta=-0,15 ; p=0,002)$. Já futuro $[\mathrm{F}(3,359)=22,17$; $p<0,001 ; \mathrm{R}=0,39)]$ teve como preditores o ambientalismo ecocêntrico $(\beta=0,31 ; p<0,001)$, o ambientalismo antropocêntrico $(\beta=0,13 ; p<0,001)$ e, negativamente, a apatia ambiental $(\beta=-0,13 ; p<0,001)$. Nessa etapa houve aumento na porcentagem de variância em ambos os modelos, tendo atingido $21 \%$ e $15 \%$ respectivamente.

Do total de participantes, 77\% (294/380) responderam afirmativamente à questão sobre a prática de cuidado ambiental e relataram algum tipo de ação. As ações mais comumente relatadas foram relativas ao manejo de resíduos (e.g., não jogar lixo no chão), à conscientização (e.g., educação ambiental) e à redução do consumo (e.g., economia de água e/ou de energia). Não foram encontradas associações significativas entre o relato da prática de cuidado ambiental e as variáveis sexo, idade e tipo de escola em que o participante estudou.

Os não-cuidadores apresentaram maiores médias em antropocentrismo e, como esperado, em apatia ambiental e imediatismo. Não houve diferenças entre os grupos quanto ao ecocentrismo. E, por fim, foram encontradas diferenças entre grupos com relação à variável sexo, tendo as mulheres apresentado maior ecocentrismo, e os homens maior apatia ambiental e imediatismo (Tabela 3).

\section{DISCUSSÃO}

No presente estudo foram investigadas relações entre indicadores de compromisso pró-ecológico e de orientação temporal e suas associações com o relato de práticas de cuidado ambiental. De forma geral, os resultados foram coerentes com estudos anteriores, aportando novas evidências empíricas relativas à pertinência de considerar a perspectiva temporal como

TABELA 3

Diferenças de médias (M) e desvios padrão (DP) entre os fatores das escalas de AEA e CCF para cuidado ambiental e sexo

\begin{tabular}{|c|c|c|c|c|c|c|c|c|c|c|c|c|}
\hline \multirow{2}{*}{ Variável } & \multirow{2}{*}{ Grupos } & \multirow{2}{*}{$n$} & \multicolumn{2}{|c|}{$A N T^{1}$} & \multicolumn{2}{|c|}{$E C O^{2}$} & \multicolumn{2}{|c|}{$A P A^{3}$} & \multicolumn{2}{|c|}{ Imediatismo } & \multicolumn{2}{|c|}{ Futuro } \\
\hline & & & $M$ & $D P$ & $M$ & $D P$ & $M$ & $D P$ & $M$ & $D P$ & $M$ & $D P$ \\
\hline \multirow[t]{2}{*}{ Cuidado ambiental } & Cuidadores & 294 & $3,68^{*}$ & 0,95 & 5,11 & 1,21 & $2,39 *$ & 0,85 & $1,38^{*}$ & 0,54 & 2,96 & 0,54 \\
\hline & Não-cuidadores & 86 & $4,04 *$ & 0,89 & 4,85 & 1,37 & $2,82 *$ & 1,16 & $1,64 *$ & 0,73 & 2,88 & 0,60 \\
\hline \multirow[t]{2}{*}{ Sexo } & Mulheres & 291 & 3,78 & 0,93 & $5,18 *$ & 1,15 & $2,33^{*}$ & 0,86 & $1,39 *$ & 0,59 & 2,95 & 0,56 \\
\hline & Homens & 89 & 3,69 & 1 & $4,62 *$ & 1,45 & $2,98 *$ & 1,01 & $1,60 *$ & 0,61 & 2,95 & 0,55 \\
\hline
\end{tabular}

${ }^{1}$ Antropocentrismo; ${ }^{2}$ Ecocentrismo; ${ }^{3}$ Apatia ambiental.

${ }^{*} p \leq 0,05$. 
uma das dimensões envolvidas na adoção (ou não) de uma postura em favor do meio ambiente, quando considerada a noção de sustentabilidade. A relevância do estudo também decorre do fato de que os estudos sobre essas relações tem sido realizados em sua maioria em contexto internacional.

Em consonância com a literatura, ainda que com índices estatísticos modestos, foram encontradas evidências empíricas das associações entre compromisso pró-ecológico, mais fortemente o ecocentrismo, e a orientação temporal de futuro (Barros, 2011; CorralVerdugo, 2010; Gifford et al., 2009; Joireman, 2005; McElwee \& Brittain, 2009; Milfont \& Gouveia, 2006; Milfont, Wilson \& Diniz, 2012; Pinheiro \& CorralVerdugo, 2010).

Entretanto, houve uma ambiguidade quanto às relações entre antropocentrismo e as orientações temporais, visto que tal postura apresentou correlação positiva tanto com futuro, quanto com imediatismo. Tal evidência sugere que, além da diferença existente entre as bases valorativas das posturas antropocêntrica e ecocêntrica, apontada tanto no estudo original da escala quanto em estudos posteriores (Coelho, Gouveia \& Milfont, 2006; Schultz \& Zelezny, 1999; Thompson \& Barton, 1994), a orientação temporal pode também ser considerada como variável que diferencia tais posturas. É válido aprofundar o entendimento sobre a orientação temporal de pessoas antropocêntricas, visto que tal orientação esteve associada à ausência de cuidado ambiental.

Ressalta-se, ainda, a relevância da escala de apatia ambiental no contexto de mensuração do compromisso pró-ecológico, visto que resultou num contraponto importante quando consideradas as associações com as demais variáveis, especialmente com a orientação temporal. Nesse sentido, recomenda-se que estudos posteriores considerem medir a postura de indiferença quanto às questões ambientais, aprofundando sua relação com o imediatismo, assim como identificando associações com outras variáveis.

Quando considerado o relato de práticas de cuidado ambiental, o ecocentrismo e a orientação de futuro não diferenciaram os grupos. Houve associações evidentes entre o antropocentrismo, a apatia ambiental e o imediatismo e a ausência de práticas de cuidado ambiental. O que reforça a ideia de que é necessário explorar as dimensões psicológicas associadas com o não engajamento em práticas de cuidado ambiental.

Estudos posteriores podem suplantar algumas das lacunas apontadas na presente investigação abordando novos grupos de participantes, com características diferentes e em contextos de coleta diferenciados. Tendo em vista que a maior parte dos estudos que abordam as mesmas relações aqui exploradas foram realizadas em outros países, observa-se que a manipulação de novas variáveis, inclusive novas variáveis sociodemográficas a partir de amostras mais heterogêneas, pode enriquecer e produzir avanços no conhecimento acerca da adoção de estilos de vida sustentáveis em contexto brasileiro.

\section{REFERÊNCIAS}

Arnocky, S., Milfont, T. L., \& Nicol, J. R. (2014). Time Perspective and Sustainable Behavior: Evidence for the Distinction Between Consideration of Immediate and Future Consequences. Environment and Behavior, 46(5), 556-582. doi:10.1177/0013916512474987

Azevedo, P. K. U. (2008). Hetero-avaliação do comprometimento pró-ambiental de colegas de turma do Curso de Controle Ambiental do CEFET/RN e sua relação com grau de envolvimento em práticas pró-ambientais. Painel apresentado no XIX Congresso de Iniciação Científica da Universidade Federal do Rio Grande do Norte, Natal.

Coelho, A., Gouveia, V., \& Milfont, T. L. (2006). Valores humanos como explicadores de atitudes ambientais e intenção de comportamento pró-ambiental. Psicologia em estudo, 11(1), 199-207. doi: 10.1590/S1413-73722006000100023

Comissão Mundial sobre Meio Ambiente e Desenvolvimento (1991). Nosso Futuro Comum. Rio de Janeiro: Editora da Fundação Getúlio Vargas.

Corral-Verdugo, V. (2010). Psicologia de la Sustentabilidad: un análisis de lo que nos hace pro-ecológicos y pro-sociales. Cidade do México: Trillas.

Corral-Verdugo, V. \& Pinheiro, J. Q. (2004). Aproximaciones al estudio de la conducta sustentable. Medio Ambiente $y$ Comportamiento Humano, 5(1/2), 1-26.

Dancey, C. P. \& Reidy, J. (2006). Estatística sem matemática para psicologia. Porto Alegre: Artmed.

Dietz, T., Fitzgerald, A., \& Shwom, R. (2005). Environmental Values. Annual Review of Environment and Resources, 30(1), 335-372. doi:10.1146/annurev.energy.30.050504.144444

Enzler, H. (2013). Consideration of Future Consequences as a Predictor of Environmentally Responsible Behavior: Evidence From a General Population Study. Environment and Behavior, 28. doi:10.1177/0013916513512204

García-Mira, R. \& Marcote, P. V. (2009). Sostenibilidad, valores y cultura ambiental. Madri: Ediciones Piramide.

Gifford. R., Scannell, L., Kormos, C., Smolova, L., Biel, A., Boncu, S., et al. (2009). Temporal pessimism and spatial optimism in environmental assessments: An 18-nation study. Journal of Environmental Psychology, 29(1), 1-12. doi: 10.1016/j. jenvp.2008.06.001

Groot, J. I. M. \& Steg, L. (2010). Relationships between value orientation, self-determinated motivational types and proenvironmental behavioural intentions. Journal of Environmental Psychology, 30(4), 368-378. doi: 10.1016/j.jenvp.2010.04.002

Gurgel, F. F. \& Pinheiro, J. Q. (2011). Compromisso pró-ecológico. In S. Cavalcante \& G. A. Elali. Temas básicos em psicologia ambiental (pp. 159-173). Petrópolis: Vozes.

Joireman, J. (2005). Environmental problems as social dilemmas: the temporal dimension. In A. Strathman \& J. Joireman (Orgs.). Understanding behavior in the context of time (pp. 289-304). Mahwah, New Jersey: Erlbaum. 
Joireman, J., Balliet, D., Sprott, D., Spangenberg, E., \& Schultz, J. (2008). Consideration of future consequences, ego-depletion, and self-control: Support for distinguishing between CFC-Immediate and CFC-Future sub-scales. Personality and Individual Differences, 45(1), 15-21. doi: 10.1016/j.paid.2008.02.011

Joireman, J., Shaffer, M. J., Balliet, D., \& Strathman, A. (2012). Promotion orientation explains why future-oriented people exercise and eat healthy: evidence from the twofactor consideration of future consequences-14 scale. Personality \& social psychology bulletin, 38(10), 1272-1287. doi:10.1177/0146167212449362

Kazdin, A. E. (2009). Psychological science's contributions to a sustainable environment: extending our reach to a grand challenge of society. American Psychologist, 64(5), 339-356. doi: $10.1037 / \mathrm{a} 0015685$

McElwee, R. O. \& Brittain, L. (2009). Optimism for the world's future versus the personal future: application to environmental attitudes. Current Psychology, 28(2), 133-145. doi: 10.1007/ s12144-009-9051-4

Milfont, T. L. \& Gouveia, V. V. (2006). Time perspective and values: An exploratory study of their relations to environmental attitudes. Journal of Environmental Psychology, 26(1), 71-82.

Milfont, T. L. \& Duckitt, J. (2010). The environmental attitudes inventory: A valid and reliable measure to assess the structure of environmental attitudes. Journal of Environmental Psychology, 30(1), 80-94. doi:10.1016/j.jenvp.2009.09.001

Milfont, T. L., Wilson, J., \& Diniz, P. (2012). Time perspective and environmental engagement: A meta-analysis. International journal of psychology, 47(5), 325-334. doi: 10.1080/ 00207594.2011.647029

Pato, C. M. L. \& Tamayo, A. (2006). A Escala de Comportamento Ecológico: desenvolvimento e validação de um instrumento de medida. Estudos de Psicologia, 11(3), 289-296.

Petrocelli, J. V. (2003). Factor validation of the consideration of future consequences scale: evidence for a short version. Journal of Social Psychology, 143(4), 405-413. doi: 10.1080/ 00224540309598453

Pinheiro J. Q. \& Pinheiro, T. F. (2007). Cuidado ambiental: Ponte entre psicologia e educação ambiental? Psico, 38(1), 25-34.

Pinheiro, J. Q. \& Corral-Verdugo, V. (2010). Time perspective and sustainable behavior. In V. Corral-Verdugo, C. H. García-Cadena, \& M. Frías-Armenta (Orgs.). Psychological approaches to sustainability. Current trends in theory, research and applications (pp. 205-224). Hauppauge, Nova York: Nova Science.

Pessoa, V. S. \& Pinheiro, J. Q. (2010). "Do que Você Lembra quando Pensa em Energia do Vento?" Um Estudo sobre o Conhecimento da Energia Eólica, Revista Interamericana de Psicologia, 44(2), 361-367.
Pol, E. (2002). The theoretical background of the City-IdentitySustainability Network. Environment and Behavior, 34(1), 8-25. doi: 10.1177/0013916502034001002

Rappange, D., Brouwer, W., \& Van Exel, N. J. A. (2009). Back to the consideration of future consequences scale: Time to reconsider? Journal of Social Psychology, 149(5), 562-584. doi: $10.1080 / 00224540903232324$

Schultz, P. W. \& Zelezny, L. (1999). Values as predictors of environmental attitudes: evidence for consistency across 14 coutries. Journal of Environmental Psychology, 19(3), 255-265. doi: 10.1006/jevp.1999.0129

Sommer, B. \& Sommer, R. (1997). A practical guide to behavioral research ( $4^{\mathrm{a}}$ ed.). Nova York: Oxford University Press.

Sousa, A. F. (2009). Percepção social do compromisso ambiental de adolescentes e sua relação com outros indicadores de próambientalidade. Relatório final de bolsista de iniciação científica apresentado à Pró-Reitoria de Pesquisa da Universidade Federal do Rio Grande do Norte, Natal.

Strathman, A., Gleicher, F., Boninger, D. S., \& Edwards, C. S. (1994). The consideration of future consequences: Weighing immediate and distant outcomes of behavior. Journal of Personality and Social Psychology, 66(1), 742-752. doi: 10.1037/0022-3514.66.4.742

Toepoel, V. (2010). Is consideration of future consequences a changeable construct? Personality and Individual Differences, 48(8), 951-956. doi: 10.1016/j.paid.2010.02.029

Thompson, S. C. G., \& Barton, M. (1994). Ecocentric and anthropocentric attitudes toward the environment. Journal of Environmental Psychology, 14(2), 149-157. doi: 10.1016/ S0272-4944(05)80168-9

Zimbardo, P. G., \& Boyd, J. N. (1999). Putting time in perspective: A valid, reliable individual-differences metric. Journal of Personality and Social Psychology, 77(6), 1271-1288. doi: 10.1037/0022-3514.77.6.1271

Autores:

Raquel F. Diniz - Doutoranda, Universidade Federal do Rio Grande do Norte. José Q. Pinheiro - Doutor, Universidade Federal do Rio Grande do Norte.

Endereço de correspondência:

Raquel F. Diniz

José Simões de Araújo, 967

CEP 58035-070 João Pessoa, PB, Brasil

E-mail: raquelfdiniz@gmail.com

Recebido em: 16.05 .2014

Aceito em: 10.08.2014 